\title{
ВЗАИМОСВЯЗЬ ЦЕННОСТЕЙ И СОЦИАЛЬНО-ЭКОНОМИЧЕСКИХ ПРЕДСТАВЛЕНИЙ У КОРЕЙСКИХ И РОССИЙСКИХ СТУДЕНТОВ
}

\author{
Г. ЦОЙ ${ }^{\mathrm{a}}$ Н.М. ЛЕБЕДЕВА
}

${ }^{a}$ Национальньй исследовательский университет «Высшая школа экономики», 101000, Россия, Москва, ул. Мясницкая, д. 20

\section{Резюме}

В статье рассматриваются результаты изучения взаимосвязи ценностей и социально-экономических представлений корейских и российских студентов. Теоретическими основаниями исследования послужили выводы российских и зарубежных авторов в области социальной, экономической и кросс-культурной психологии, а также институциональной и поведенческой экономики. Для изучения ценностей использовалась теория индивидуальных ценностей Ш. Шварца (Schwartz et al., 2012), а также ряд работ, посвященных рассмотрению взаимосвязи культуры и экономики (Weber, 1978; Hofstede, 1980; Furnham, 1984; Inglehart, 1997; Журавлев, Купрейченко, 2007; Лебедева, Татарко, 2011). Цель исследования состояла в выявлении универсальных и культурно-специфических связей между индивидуальными ценностями и социально-экономическими представлениями российских и корейских студентов. Испытуемыми были студенты в возрасте от 14 до 30 лет (157 корейцев и 211 россиян), проживающие в Сеуле (Республика Корея) и Москве (Россия) соответственно. Социально-психологический опрос проводился методом «снежного кома», содержание опросников на двух языках было идентичным. Корейская версия опросника была переведена и адаптирована в соответствии с требованиями кросс-культурной психологии - Think-aloud (Ericsson, Simon, 1980). Результаты опроса обрабатывались с помощью SPSS и приложения AMOS (эксплораторный факторный анализ и моделирование структурными уравнениями). В исследовании было показано, что существуют универсальные связи ценностей Открытость изменениям с позитивным Отношением к конкуренции и связи ценностей Самопреодоление с Удовлетворенностью материальным положением и Значимостью денег. А также выявлена культурно-специфическая связь между блоком ценностей Открытость изменениям и позитивным отношением к конкуренции у россиян и корейцев: позитивная и негативная связи соответственно. Полученные результаты и выводы исследования могут быть использованы при подготовке рекомендаций в сфере кросс-культурного бизнес-консультирования.

Ключевые слова: индивидуальные ценности, экономические представления, кросс-культурная психология.

Статья подготовлена в ходе проведения исследования в рамках Программы фундаментальных исследований Национального исследовательского университета «Высшая школа экономики» (НИУ ВШЭ) и с использованием средств субсидии в рамках государственной поддержки ведущих университетов Российской Федерации «5-100». 


\section{Введение}

Рассмотрение проблем, связанных с влиянием культурных и психологических факторов на экономическое поведение людей и социально-экономическое развитие стран, является в настоящее время чрезвычайно актуальным (Hofstede, 1980; Inglehart, 1997; Лебедева, Татарко, 2011).

Экономическое поведение личности понимается психологами как особый вид социального поведения, и интерес исследователей смещается в область понимания социально-психологических регуляторов этой области жизнедеятельности: ценностей, норм, установок, стереотипов, атрибутивных процессов (Cherrington, 1980; Bagozzi et al., 2002). Изучая нынешнее постиндустриальное общество, зарубежные и отечественные исследователи пришли к выводу, что культура влияет на экономическое поведение человека, а также на экономическое развитие в целом (Weber, 1978; Inglehart, 1997). В этой связи встает необходимость поиска культурно-универсальных и культурно-специфических факторов, влияющих на экономическое поведение. Сегодня в социальной психологии и смежных науках предложен ряд теоретико-эмпирических подходов к измерению культурно-психологических особенностей индивида (Hofstede, 1980; Inglehart, 1997; Schwartz, 2003; Leung, Bond, 2004).

С ужесточением санкций западных стран против России становится все более перспективной и крайне необходимой переориентация России на экономическое, политическое, военное, научное и культурное сотрудничество со странами Дальнего Востока -
«Разворот на Восток» (Караганов, 2014), в том числе и с Южной Кореей. В дальнейшем результаты и выводы исследования могут быть использованы при разработке рекомендации по формированию экономической политики России и Южной Кореи, а также при осуществлении сопровождения социально-экономической адаптации личности к иной социокультурной и экономической среде в данных странах.

Цель нашего поискового исследования состояла в выявлении и описании универсалий и специфики влияния ценностей на социально-экономические представления россиян и корейцев.

\section{Социально-экономические представления личности}

При рассмотрении роли сознания человека в процессе принятия экономического решения в социальной психологии используются термины «экономическое представление» и «экономические установки» как составляющие экономического сознания личности (Журавлев, Купрейченко, 2005).

Исследование психологических факторов социального и экономического поведения в зарубежной и отечественной литературе имеет большую историю. Среди зарубежных исследований можно привести кросс-культурный сравнительный анализ страхового поведения канадцев и американцев (Wharton, Harmatz, 1989), сравнительный анализ восприятия роли различных сфер услуг в США и Нидерландах (Goodwin, Verhage, 1989), сравнительное исследование отношения к налого- 
обложению в Италии и Великобритании (Lewis et al., 2009).

B России в лаборатории социальной и экономической психологии Института психологии РАН было проведено множество эмпирических исследований (Журавлев, Купрейченко, 2005, 2007) по изучению взаимосвязи экономического сознания и других социально-экономических характеристик населения, способствующих в конечном итоге развитию экономики. Особое внимание уделялось исследованию отношения личности к деньгам (Горбачева, Купрейченко, 2006).

При изучении отношения к деньгам О.С. Дейнека и другие ученые используют такие понятия, как представления о деньгах, образ денег (Дейнека, 2000), денежные аттитюды и установки (Фенько, 2000).

Результаты исследования монетарных аттитюдов, проведенных А. Фернамом, выявили шесть ортогональных факторов: зависимость, власть использование, планирование, безопасность - консерватизм, неадекватность, усилие - способность (Furnham, 1984). Автор пришел к выводу, что нерациональные потребители склонны видеть в деньгах способ решения проблем и чаще рассматривают деньги как основание для сравнения себя с другими людьми. Также они используют деньги для повышения самооценки, социального статуса, власти, при этом они, как правило, не удовлетворены своим финансовым положением.

В рамках настоящего исследования в качестве предикторов мы будем использовать набор социально-экономических представлений и монетарных аттитюдов в рамках исследовательской работы коллектива Международной научно-учебной лаборатории социокультурных исследований НИУ «Высшая школа экономики» на российской выборке: экономическая самостоятельность, экономический патернализм, удовлетворенность материальным благосостоянием, тревожность из-за денег, использование денег для управления другими людьми, сохранение-сбережение денег, контроль денег, недостаточность денег, связь действийусилий с деньгами, а также позитивное представление конкуренции, готовность к риску (Лебедева, Татарко, 2011, с. 134-150).

\section{Ценности и социально- экономические представления}

Экономическое поведение определяется не только рациональным расчетом, но и разными психологическими факторами, в числе которых находятся ценности индивидов. Ценности являются важной составляющей в содержании культуры, которая может объяснять экономическое развитие, экономические установки (Лебедева, Татарко, 2011), поведение людей в сфере экономики - отношение к труду (Schwartz, 1992).

Как отмечают Шварц и Билски, ценности являются достаточно надежным средством для определения и предсказания собственного и чужого поведения (Schwartz, Bilsky, 1990).

В своих исследованиях Ш. Шварц развил новый теоретический и методологический подход к изучению ценностей, где выделил 10 ценностей (Schwartz, 1992), которые представляют собой мотивационный континуум. Позже он предложил новый 
методологический подход к исследованию культур через призму уточненной теории базовых индивидуальных ценностей (с 19 ценностями), которые «фиксируют значимые различия мотиваций на континууме ценностей» (Schwartz et al., 2013). B нашем исследовании используются блоки ценностей и 19 ценностей уточненной теории.

Хотя ценности универсальны, ценностные приоритеты в различных культурах разные. Поэтому интересно исследовать, как связаны одинаковые ценности с одинаковыми социально-экономическими представлениями в разных культурах, в частности, в российской и корейской, так как ценностные приоритеты в этих культурах различаются.

\section{Методика}

Характеристики выборки. В исследовании приняли участие 375 респондентов. Характеристики выборки представлены в таблице 1.

Методики исследования. Для выявления индивидуальных ценностей и социально-экономических представлений московских и сеульских студентов использовались следующие методики на русском и корейском языках.
Методики оценки социально-экономических представлений личности

1. «Отношение к конкуренции» оценивалось с помощью соответствующего вопроса по 10-балльной шкале из WVS «Всемирного обзора ценностей» («Конкуренция вредна: она пробуждает у людей их худшие качества» - «Конкуренция - это хорошо. Она побуждает людей напряженно работать и развивать новые идеи»).

2. Установка на «Экономическую самостоятельность». В исследовании по 10-балльной шкале оценивались экономическая самостоятельность и патернализм, где «1»- респондент склонен к патерналистскому обществу и полностью согласен с мнением «Правительство должно нести больше ответственности за то, чтобы все граждане были обеспечены», а «10» - с мнением «Люди должны сами себя обеспечивать и не надеяться на правительство».

3. Удовлетворенность материальным положением. Данное представление измерялось по 5-балльной шкале, оценивался уровень материального благосостояния респондента («Меня устраивает уровень моего материального благосостояния»).

4. Готовность к риску. Данный показатель измерялся по 5-балльной

Половозрастной состав выборки

Таблица 1

\begin{tabular}{|l|c|c|c|c|c|c|c|c|}
\hline \multirow{2}{*}{\multicolumn{1}{r|}{ Группа }} & \multirow{2}{*}{$\mathrm{N}$} & \multicolumn{4}{|c|}{ Пол } & \multicolumn{3}{c|}{ Возраст } \\
\cline { 3 - 9 } & & муж. & $\%$ & жен. & $\%$ & $\min$ & $\max$ & $\mathrm{M}$ \\
\hline Россияне & 211 & 72 & 34.1 & 139 & 65.9 & 14 & 30 & 20.2 \\
\hline Корейцы & 157 & 71 & 45.2 & 86 & 54.8 & 17 & 30 & 23.1 \\
\hline Всего & 368 & 143 & 38.9 & 225 & 61.1 & 14 & 30 & 21.5 \\
\hline
\end{tabular}


шкале, оценивались два измерения готовности к риску:

a) психологическая готовность к риску («Люди, которые знают меня, считают, что я осторожен»);

б) готовность к риску в инвестиционном поведении («Я больше предпочитаю банковские депозиты, чем рискованные инвестиции»).

5. Установка по отношению к деньгам. В исследовании по 5-балльной шкале оценивалось отношение к деньгам с помощью девяти шкал адаптированного опросника А. Фернама (см.: Лебедева, Татарко, 2011, c. 141-143), которые в последующем факторном анализе измеряются по трем признакам: значимости, внимательному отношению и тревожности к деньгам (например, «Мои деньги это единственное, на что я могу рассчитывать»).

\section{Методика измерения индивидуаль-} ных иенностей Ш. Швариа PVQ-R (Schwartz et al., 2012)

Индивидуальные ценности рассматривались в рамках четырех блоков ценностных ориентаций: Coхранение, Открытость изменениям, Самоутверждения и Самопреодоление. Опросник на корейском языке был предоставлен автором методики Ш. Шварцем. Опросник был переведен и адаптирован психологами Католического университета Кореи (Choi, Lee, 2014).

\section{Описание результатов}

В ходе рассмотрения и изучения вопросов, связанных с социальноэкономическими представлениями, мы провели эксплораторный фак- торный анализ данных девяти шкал, определяющих отношение к богатству, и получили три фактора: «Значимость денег», «Внимательное отношение к деньгам» и «Финансовая пунктуальность» (см. таблицу 2).

Полученные факторы, характеризующие отношение к деньгам, далее будут использованы как зависимые переменные.

Графическое представление модели значимых связей блоков ценностей и социально-экономических представлений россиян и корейцев показаны на рисунках 1 и 2 соответственно. Характеристики моделей представлены в таблице 3.

Характеристики моделей показывают, что построенные модели удовлетворяют требованиям $(1<\mathrm{CMIN} / \mathrm{df}$ $<3$; CFI > .90; RMSEA < .100; PCLO$\mathrm{SE}>$.05; SRMR < .09) (Hu, Bentler, 1999), предъявляемым результатам структурного моделирования.

Результаты проведенного анализа показывают, что универсальными для данных двух групп оказываются позитивные связи блока ценностей Самоутверждения со значимостью денег и негативные - с удовлетворенностью материальным положением. Выявлена культурно-специфическая связь блока ценностей $\mathrm{Om}$ крытость изменениям и позитивного отношения к конкуренции.

\section{Обсуждение результатов}

Как уже было сказано, в связи блока ценностей Открытость изменениям с позитивным отношением к конкуренции выявлена культурная специфика: у россиян - связь позитивная, а у корейцев - негативная. Данный результат может быть про- 
Таблица 2

Результаты эксплораторного факторного анализа вопросов, направленных на оценку монетарных аттитюдов личности

\begin{tabular}{|c|c|c|c|c|c|}
\hline \multirow[b]{2}{*}{ Факторы } & \multirow[b]{2}{*}{ Утверждения } & \multicolumn{2}{|c|}{ Россияне } & \multicolumn{2}{|c|}{ Корейцы } \\
\hline & & Bec & $\%$ & $\mathrm{Bec}$ & $\%$ \\
\hline \multirow{4}{*}{$\begin{array}{l}\text { F1. } \\
\text { Значимость } \\
\text { денег }\end{array}$} & $\begin{array}{l}\text { Мои деньги - это единственное, } \\
\text { на что я могу рассчитывать }\end{array}$ & .619 & \multirow{4}{*}{$\begin{array}{c}2.43 \\
(26.95)\end{array}$} & .520 & $\begin{array}{c}2.09 \\
(23.21)\end{array}$ \\
\hline & $\begin{array}{l}\text { Деньги дают человеку большую } \\
\text { власть }\end{array}$ & .687 & & .763 & \\
\hline & $\begin{array}{l}\text { Я постоянно беспокоюсь о своем } \\
\text { финансовом положении (обр.) }\end{array}$ & -.573 & & -.622 & \\
\hline & $\begin{array}{l}\text { Ради денег я готов практически на } \\
\text { все в рамках закона }\end{array}$ & .786 & & .678 & \\
\hline \multirow{2}{*}{$\begin{array}{l}\text { F2. } \\
\text { Внимательное } \\
\text { отношение } \\
\text { к деньгам }\end{array}$} & $\begin{array}{l}\text { Я всегда знаю почти до копейки, } \\
\text { сколько денег у меня в кошельке } \\
\text { или в кармане }\end{array}$ & .754 & \multirow{2}{*}{$\begin{array}{c}1.51 \\
(16.72)\end{array}$} & .852 & $\begin{array}{c}1.51 \\
(16.75)\end{array}$ \\
\hline & $\begin{array}{l}\text { Я всегда знаю, сколько денег лежит } \\
\text { на моем счете в банке }\end{array}$ & .853 & & .879 & \\
\hline $\begin{array}{l}\text { F3. } \\
\text { Финансовая } \\
\text { тревожность }\end{array}$ & $\begin{array}{l}\text { Я часто испытываю затруднения, } \\
\text { принимая решения о трате денег } \\
\text { независимо от суммы }\end{array}$ & .735 & $\begin{array}{c}1.08 \\
(12.00)\end{array}$ & .799 & $\begin{array}{c}1.32 \\
(14.67)\end{array}$ \\
\hline
\end{tabular}

Примечание. Вес - вес утверждения, \% - процент объясняемой дисперсии и вес фактора.

Таблица 3

Показатели соответствия модели: влияние блоков ценностей на социально-экономические представления для россиян и корейцев

\begin{tabular}{|c|c|c|c|c|c|c|c|}
\hline Группа & $\chi^{2}$ & df & CMIN/df & CFI & RMSEA & PCLOSE & SRMR \\
\hline Россияне & 43.28 & 23 & 1.882 & .912 & .065 & .191 & .0619 \\
\hline Корейцы & 17.54 & 13 & 1.349 & .961 & .047 & .483 & .0680 \\
\hline
\end{tabular}

интерпретирован следующим образом. Корея - это коллективистская культура, в которой каждый должен идеально вписаться в матрицу правил, норм и законов, и, как правило, если кореец попытается пойти про- тив общества, то он станет его изгоем (Choi, 2001), т.е. корейцы более подвержены конформизму и ни при каких обстоятельствах не должны стремиться к самостоятельности. 
Рисунок 1

Модель влияния блоков ценностей на социально-экономические представления в группе россиян

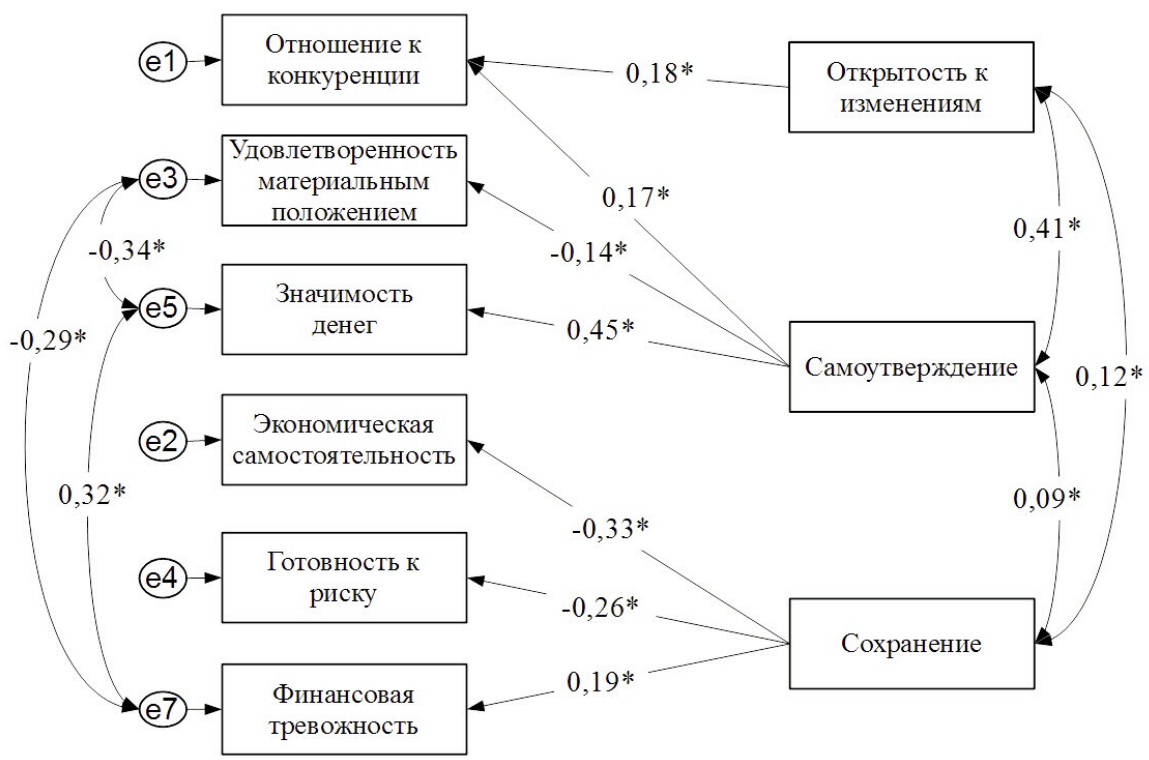

Рисунок 2

Модель влияния блоков ценностей на социально-экономические представления в группе корейцев

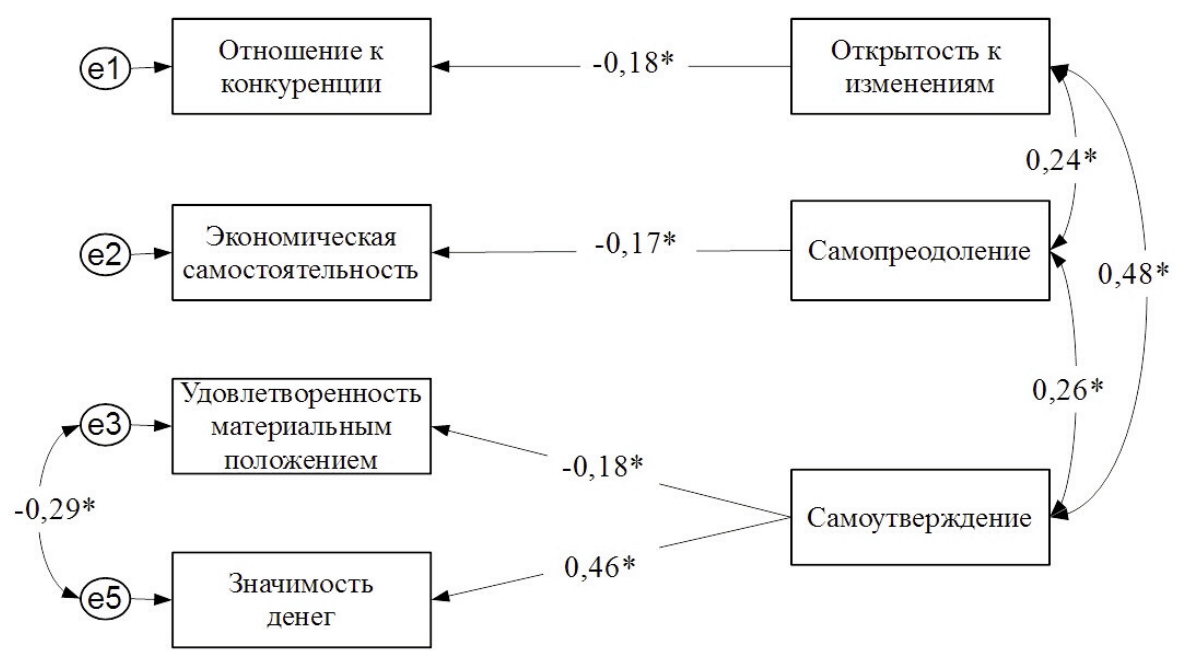


Что касается влияния блока ценностей Самоутверждение, то здесь ценности оказывают положительное влияние на значимость денег и негативное - на удовлетворенность материальным положением в обеих группах. Это значит, что среди людей, принадлежащих к этим обществам, распространено представление о том, что для занятия высокой позиции в обществе необходимы власть и деньги, что может стать объяснением первой связи. Также при стремлении к власти и успеху людям нужны дополнительные усилия и средства: повышение квалификации, средства для инвестиций и т.д. В модели для группы корейцев блок ценностей Самопреодоление показал негативную связь с установкой на экономическую самостоятельность. Это может быть обусловлено особенностями мировоззрения конфуцианской Кореи, где стремление к богатству считается несовместимым с ценностями, входящими в блок Самопреодоление (благожелательность - чувство долга, благожелательность - забота, универсализм забота о других, универсализм забота о природе и универсализм толерантность). Кроме того, возможно, что в нашей выборке корейцы, являющиеся студентами, пока еще не вступили в самостоятельную экономическую жизнь, для них достижение материального благополучия и среднего уровня жизни невозможно без опеки государства.

Значимых связей между блоком ценностей Сохранение и экономическими представлениями у корейцев не обнаружено. Ценности данного блока показали значимую связь с экономической самостоятельностью, готовностью к риску и финансовой тревожностью у россиян. Российские студенты оказались более склонны к патернализму, более обеспокоены финансовым состоянием и меньше готовы рисковать.

\section{Выводы}

Были выявлены связи ценностей и характеристик экономического поведения в обеих группах.

- В группе россиян блок ценностей Открытость к изменениям положительно связан с позитивным отношением к конкуренции. Ценности Самоутверждения положительно связаны со значимостью денег и позитивным отношением к конкуренции, отрицательно - с удовлетворенностью материальным положением. Ценности Сохранения имеют положительную связь с финансовой тревожностью, негативную связь с готовностью к риску и экономической самостоятельностью.

- В группе корейцев блок ценностей Открытость к изменениям имеет лишь единственную негативную связь с позитивным отношением к конкуренции. Блок ценностей Самоутверждение позитивно связан со значимостью денег и негативно с удовлетворенностью материальным положением. Блок ценностей Самопреодоление негативно связан с экономической самостоятельностью.

Была выявлена культурно-специфическая связь позитивного отношения к конкуренции и блока ценностей Открытость изменениям. 


\section{Литература}

Горбачева, Е. И., Купрейченко, А. Б. (2006). Отношение личности к деньгам: нравственные противоречия в оценках и ассоциациях. Психологический журнал, 27(4), 26-37.

Дейнека, О. С. (2000). Экономическая психология. СПб.: Изд-во Санкт-Петербургского университета.

Журавлев, А. Л., Купрейченко, А. Б. (2005). Проблемы экономической психологии. М.: Издательство «Институт психологии РАН».

Журавлев, А. Л., Купрейченко, А. Б. (2007). Экономическое самоопределение: Теория и эмпирические исследования. М.: Издательство «Институт психологии РАН».

Караганов, С. (2014, 26 августа). Вперед к Великому океану. Российская газета, с. 4. Режим доступа: http://rg.ru/2014/08/26/usilenie.html

Лебедева, Н. М., Татарко, А. Н. (2011). Ценности культуры и модели экономического поведения. М.: Спутник.

Фенько, А. Б. (2000). Проблема денег в зарубежных психологических исследованиях. Психологический журнал, 21(1), 50-62.

Bagozzi, R., Gurhan-Canli, Z., \& Priester, J. (2002). The social psychology of consumer behaviour. Buckingham, UK: Open University Press.

Cherrington, D. J. (1980). The work ethic. New York: American Management Association.

Choi, K. W. (2001). A merged government ministry through organizational culture: A interpretative approach based on schema. Review of Public Administration, 35(3), 127-145. (на кор. яз.)

Choi, J. W., \& Lee, Y. H. (2014). Validation of the Korean version of the Portrait Values Questionnaire-Revised (PVQ-R). The Korean Journal of Psychology, 33(3), 553-593.

Ericsson, K. A., \& Simon, H. A. (1980). Verbal reports as data. Psychological review, 87(3), 215-251.

Furnham, A. (1984). Many sides of the coin: The psychology of money usage. Personality and Individual Differences, 5(5), 501-509.

Goodwin, C., \& Verhage, B. J. (1989). Role perceptions of services: a cross-cultural comparison with behavioral implications. Journal of Economic Psychology, 10(4), 543-558.

Hofstede, G. (1980). Culture's consequences: International differences in work-related values. Beverly Hills, CA: Sage.

Hu, L. T., \& Bentler, P. M. (1999). Cutoff criteria for fit indexes in covariance structure analysis: Conventional criteria versus new alternatives. Structural Equation Modeling: A Multidisciplinary Journal, 6(1), 1-55.

Inglehart, R. (1997). Modernization and postmodernization: Cultural, economic, and political change in 43 societies (Vol. 19). Princeton, NJ: Princeton University Press.

Leung, K., \& Bond, M. H. (2004). Social axioms: A model for social beliefs in multicultural perspective. Advances in Experimental Social Psychology, 36, 119-197.

Lewis, A., Carrera, S., Cullis, J., \& Jones, P. (2009). Individual, cognitive and cultural differences in tax compliance: UK and Italy compared. Journal of Economic Psychology, 30(3), 431-445.

Schwartz, S. H. (1992). Universals in the content and structure of values: Theoretical advances and empirical tests in 20 countries. Advances in Experimental Social Psychology, 25(1), 1-65.

Schwartz, S. H. (2003). A proposal for measuring value orientations across nations. In Questionnaire Development Package of the European Social Survey (pp. 259-319).

Schwartz, S. H. (2014). Rethinking the concept and measurement of societal culture in light of empirical findings. Journal of Cross-Cultural Psychology, 45(1), 5-13. 
Schwartz, S. H., \& Bilsky, W. (1990). Toward a theory of the universal content and structure of values: Extensions and cross-cultural replications. Journal of Personality and Social Psychology, 58(5), 878.

Weber, M. (1978). Economy and society: An outline of interpretive sociology. Berkeley, CA: University of California Press.

Wharton, J. D., \& Harmatz, H. R. (1989). An exploratory investigation of how cultural attitudes relate to life insurance holdings: A cross-cultural comparison. Journal of Economic Psychology, 10(2), $217-227$
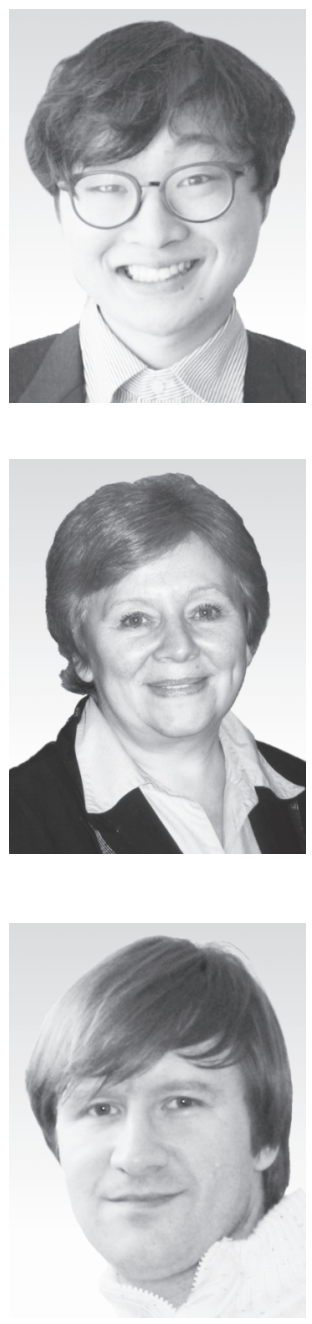

Цой Гунвон - аспирант, департамент психологии, факультет социальных наук, Национальный исследовательский университет «Высшая школа экономики».

Сфера научных интересов: ценности, экономические представления, адаптация.

Контакты: gchoi@hse.ru

Лебедева Надежда Михайловна - профессор, департамент психологии, факультет социальных наук, и заведующая лабораторией, Международная научно-учебная лаборатория социокультурных исследований, Экспертный институт, Национальный исследовательский университет «Высшая школа экономики», доктор психологических наук.

Контакты: nlebedeva@hse.ru

Татарко Александр Николаевич - доцент, департамент психологии, факультет социальных наук, ведущий научный сотрудник, Международная научно-учебная лаборатория социокультурных исследований, Экспертный институт, Национальный исследовательский университет «Высшая школа экономики», доктор психологических наук.

Контакты: tatarko@yandex.ru 


\title{
The Relationship between Values and Socio-Economic Attitudes among Korean and Russian Students
}

\author{
K. Choi ${ }^{\mathrm{a}}$, N.M. Lebedeva ${ }^{\mathrm{a}}$, A.N. Tatarko ${ }^{\mathrm{a}}$ \\ ${ }^{a}$ National Research University Higher School of Economics, 20 Myasnitskaya str., Moscore, 101000, \\ Russian Federation
}

\begin{abstract}
The article reviews the results of the study on the interaction of values and socio-economic views of Korean and Russian students. The theoretical basis for the research is the works by Russian and foreign authors in social, economic and cross-cultural psychology, as well as in institutional and behavioral economy. The theory of individual values by S. Schwartz was used for the study on values (Schwartz et al., 2012), as well as some works on interaction of culture and economy (Weber, 1978; Hofstede, 1980; Furham, 1984; Inglehart, 1997; Zhuravlev, Kupreychenko, 2007; Lebedeva, Tatarko, 2011). The objective of the research was the detection of universal and culture-specific correlations between individual values and socio-economic views of Russian and Korean students. The subjects were students of 14 to 30 years of age (157 Koreans and 211 Russians), living in Seoul (Republic of Korea) and Moscow (Russia) correspondingly. Socio-psychological survey was administered with the method of "snowball", the content of the questionnaires in two languages was identical. The Korean version of the questionnaire was translated and adapted in accordance with the requirements of cross-cultural psychology - Think-aloud (Ericsson \& Simon, 1980; Someren, Barnard, Sandberg, 1994). The results of the survey were calculated with SPSS and AMOS (exploratory factor analysis and structural equation modeling). The research showed that there are universal correlations of values Openness to Change with Positive attitude toward competition and between Self-transcendence and Satisfaction with material status and Importance of money. Also cultural-specific correlation was found between the block of values Openness to Change and Positive attitude toward competition in Russians and Koreans - which was positive and negative correspondingly. The acquired results and findings of the research can be used in drafting of recommendations in the sphere of crosscultural business-consulting.
\end{abstract}

Keywords: individual values, economic attitudes, cross-cultural psychology.

\section{References}

Bagozzi, R., Gurhan-Canli, Z., \& Priester, J. (2002). The social psychology of consumer behaviour. Buckingham, UK: Open University Press.

Cherrington, D. J. (1980). The work ethic. New York: American Management Association.

Choi, K. W. (2001). A merged government ministry through organizational culture: A interpretative approach based on schema. Review of Public Administration, 35(3), 127-145. (на кор. яз.).

Choi, J. W., \& Lee, Y. H. (2014). Validation of the Korean version of the Portrait Values Questionnaire-Revised (PVQ-R). The Korean Journal of Psychology, 33(3), 553-593. 
Deineka, O. S. (2000). Ekonomicheskaya psikhologiya [Economic psychology]. Saint Petersburg: Saint Petersburg University Press.

Ericsson, K. A., \& Simon, H. A. (1980). Verbal reports as data. Psychological Review, 87(3), 215-251.

Furnham, A. (1984). Many sides of the coin: The psychology of money usage. Personality and Individual Differences, 5(5), 501-509.

Goodwin, C., \& Verhage, B. J. (1989). Role perceptions of services: a cross-cultural comparison with behavioral implications. Journal of Economic Psychology, 10(4), 543-558.

Gorbacheva, E. I., \& Kupreychenko, A. B. (2006). The attitude of the person to money: Moral contradictions in estimations and associations. Psikhologicheskii Zhurnal, 27(4), 26-37.

Hofstede, G. (1980). Culture's consequences: International differences in work-related values. Beverly Hills, CA: Sage.

Hu, L. T., \& Bentler, P. M. (1999). Cutoff criteria for fit indexes in covariance structure analysis: Conventional criteria versus new alternatives. Structural Equation Modeling: A Multidisciplinary Journal, 6(1), 1-55.

Inglehart, R. (1997). Modernization and postmodernization: Cultural, economic, and political change in 43 societies (Vol. 19). Princeton, NJ: Princeton University Press.

Karaganov, S. (2014, August 26). Vpered k Velikomu okeanu [Ahead to the Great Ocean]. Rossiiskaya Gazeta, p. 4. Retrieved from http://rg.ru/2014/08/26/usilenie.html

Lebedeva, N. M., \& Tatarko, A. N. (2011). Tsennosti kul'tury i modeli ekonomicheskogo povedeniya [Cultural values and models of economic behavior]. Moscow: Sputnik.

Leung, K., \& Bond, M. H. (2004). Social axioms: A model for social beliefs in multicultural perspective. Advances in Experimental Social Psychology, 36, 119-197.

Lewis, A., Carrera, S., Cullis, J., \& Jones, P. (2009). Individual, cognitive and cultural differences in tax compliance: UK and Italy compared. Journal of Economic Psychology, 30(3), 431-445.

Schwartz, S. H. (1992). Universals in the content and structure of values: Theoretical advances and empirical tests in 20 countries. Advances in Experimental Social Psychology, 25(1), 1-65.

Schwartz, S. H. (2003). A Proposal for Measuring Value Orientations across Nations. Chapter 7 in the Questionnaire Development Package of the European Social Survey (pp. 259-319).

Schwartz, S. H. (2014). Rethinking the concept and measurement of societal culture in light of empirical findings. Journal of Cross-Cultural Psychology, 45(1), 5-13.

Schwartz, S. H., \& Bilsky, W. (1990). Toward a theory of the universal content and structure of values: Extensions and cross-cultural replications. Journal of Personality and Social Psychology, 58(5), 878.

Weber, M. (1978). Economy and society: An outline of interpretive sociology. Berkeley: University of California Press.

Wharton, J. D., \& Harmatz, H. R. (1989). An exploratory investigation of how cultural attitudes relate to life insurance holdings: A cross-cultural comparison. Journal of Economic Psychology, 10(2), 217-227.

Zhuravlev, A. L., \& Kupreychenko, A. B. (2005). Problemy ekonomicheskoi psikhologii [Issues of economic psychology]. Moscow: Institute of Psychology of Russian Academy of Sciences.

Zhuravlev, A. L., \& Kupreychenko, A. B. (2007). Ekonomicheskoe samoopredelenie: Teoriya i empiricheskie issledovaniya [Economic self-determination: Theory and empirical research]. Moscow: Institute of Psychology of Russian Academy of Sciences. 
Keunwon Choi - postgraduate student, School of Psychology, Faculty of Social Sciences, National Research University Higher School of Economics.

Research area: values, economic representation, adaptation.

E-mail: gchoi@hse.ru

Nadezhda M. Lebedeva - professor, Faculty of Social Sciences, School of Psychology, laboratory head, International Scientific-Educational Laboratory for Socio-Cultural Research, Expert Institute, National Research University Higher School of Economics, D.Sc.

E-mail: nlebedeva@hse.ru

Alexander N. Tatarko - associate professor, Faculty of Social Sciences, School of Psychology, senior researcher, International Laboratory for Socio-Cultural Research, National Research University Higher School of Economics, D.Sc.

E-mail: tatarko@yandex.ru 